\section{Guiding New Generations for Decades}

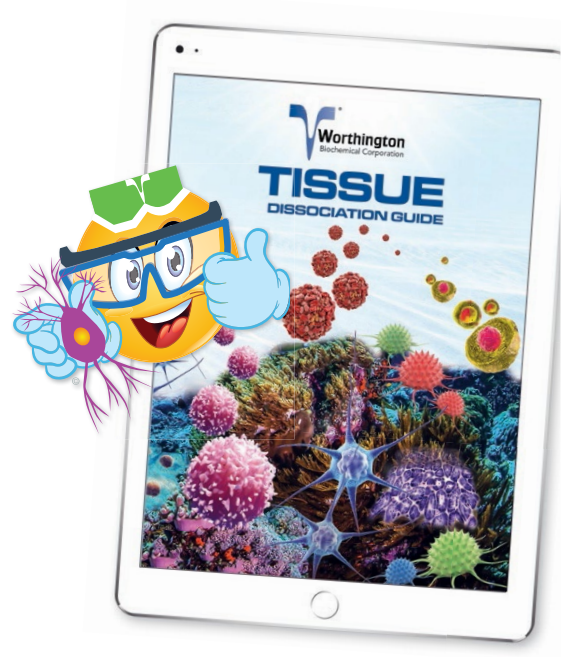

Worthington is supporting life science research and discovery, and we have done so for over 70 years.

Authoring technical manuals and guides both in print and digital formats. And, we are cited in thousands of respected scientific journals across the globe.

\section{Sharing Our Enzyme Expertise}

\section{Order your free copy of the NEW Tissue Dissociation Guide, 18th Edition. Simply go to:}

\section{Worthington-Biochem.com/ TissueDissociation/default.html}

\section{Come see us at SFN- Booth 2136}

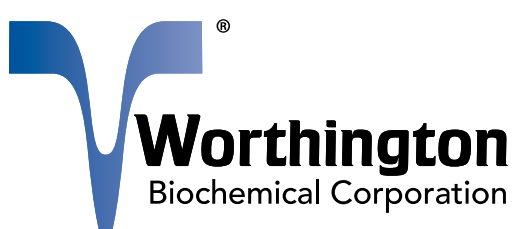

$800.445 .9603 \bullet 732.942 .1660$

\title{
A tissue-to-organelle view of cellular proteins
}

By coupling multiplex iterative indirect immunofluorescence imaging with computer vision methods, researchers can detect at least 40 different proteins with subcellular resolution.

C ells continually receive and respond to a variety of cues, which are integrated to help the cells adjust their shape, size, and function. Such cellular plasticity would be impossible without the proteins within and around the cell, whose joint actions instruct the cell on how to act.

Recent efforts have cataloged and described proteins' subcellular distribution in single cells, but how these complex snapshots of cellular proteins are functionally linked to a cell's phenotypic state, microenvironment, and response to varying conditions is still unclear. To address this issue, a team led by Lucas Pelkmans from the University of Zürich developed a method that can be used to visualize 40 different proteins and their posttranslational modifications in an individual tissue cell, with subcellular resolution.

The approach consists of two parts: multiplex iterative indirect immunofluorescence imaging (4i), which provides a host of high-resolution images of 40 protein stainings, and an unsupervised computer vision approach to quantify protein organization with submicrometer spatial detail in hundreds of single cells. The final result is a comprehensive multiplex protein map (MPM) of multiple cells in a dish with subcellular resolution. "It's a bit like Google Earth," Pelkmans says. "You can stitch all the images together, make a big overview, zoom out and look at patterns. But because the images were generated at high resolution, you can then also zoom in to a very high level of detail."

A major obstacle in 4i development was photo-cross-linking of antibodies to the sample during imaging. The complete removal of antibodies after staining is key for the success of subsequent stainings. But the removal of photo-cross-linked antibodies requires harsh elution buffers that also disturb cellular ultrastructure, thus decreasing the effectiveness of the next staining. The team solved this issue by adding chemicals to the staining buffer that prevent antibody photocross-linking, thereby enabling thorough removal of the antibodies after each staining and preserving cell ultrastructure. This protocol allowed for 21 iterative stainings.

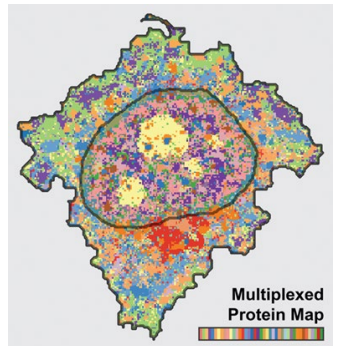

A multiplexed protein map. Adapted with permission from Gut et al. (2018)

Pelkmans predicts that in the future it might be possible to generate 150-plex protein readouts by increasing the number of antibodies that allow for direct and indirect stainings.

Finally, a custom-made unsupervised computer vision method is used to quantify properties visible both at low magnification (at the population scale) and on the subcellular scale, generating MPMs. MPMs comprehensively quantify intracellular protein composition with great spatial detail in large numbers of single cells, and are not confounded by the geometry and orientation of a cell.

The authors used the $4 \mathrm{i}$ approach to analyze the MPMs in different phases of the cell cycle, in response to cell crowding, inhibitors, and different growth conditions. In each instance, they were able to comprehensively quantify protein subcompartmentalization and systematically define cellular states, which could not be detected by multiplexed wholecell measurements.

Pelkmans hopes that the new method will advance research on drug testing, organoids, diagnostics, and precision medicine.

\section{Vesna Todorovic}

Published online: 1 October 2018 https://doi.org/10.1038/s41592-018-0163-3

Research papers

Gut, G., Herrmann, M. D. \& Pelkmans, L. Multiplexed protein maps link subcellular organization to cellular states. Science 361, eaar7042 (2018). 